\title{
Características clínicas, histopatológicas y terapéuticas del cáncer de tiroides en Colombia: serie de 1.096
} pacientes

\author{
Clinical, Histopathological and Therapeutic Characteristics of Thyroid \\ Cancer in Colombia: A case series study including 1.096 patients
}

Edwin Antonio Wandurraga Sánchez $z^{1}$ Lisseth Fernanda Marín Carrillo ${ }^{2}$, Annie Katherine Natera $M^{M e l o}{ }^{3}$, Claudia Milena Gómez Giraldo ${ }^{4}$, Freddy Niño Prato ${ }^{5}$, Henry Mauricio Arenas Quintero 6 , Rafael Castellanos Bueno ${ }^{7}$, Néstor Alfonso López Pompey, Darío Arturo De La Portilla Maya, Karen Milena Fériz Bonelo ${ }^{10}$, Alejandro Pinzón Tovar ${ }^{11}$, Juan Pablo Dueñas Muñoz $z^{12}$, Alin Abreu Lomba $^{13}$, Luis Felipe Fierro Maya ${ }^{14}$, Juan Bernardo Pinzón Barco ${ }^{15}$, José Luis Torres Grajales ${ }^{16}$, Andrés Felipe Palacio Barrientos ${ }^{17}$, Liseth Sánchez Orduz ${ }^{18}$, Rafael Eduardo García Rey ${ }^{19 \dagger}$.

${ }^{1}$ MD, Endocrinólogo, MSc Oncología Molecular. Profesor Asociado Universidad Autónoma de Bucaramanga, UNAB. Universidad Autónoma Bucaramanga. Endocrinólogos del Oriente (ENDORIENTE) FOSCAL Internacional, Floridablanca, Santander, Colombia.edwinwandurraga@gmail.com ${ }^{2} \mathrm{MD}$, Endocrinóloga. Centro Médico Carlos Ardila Lulle. Fundación Cardiovascular de Colombia. Floridablanca, Santander, Colombia.lissmarin87@gmail.com

${ }^{3} \mathrm{MD}$, Internista, Fellow de Hematooncología Hospital Militar Central.Bogotá, Colombia. anniekn86@hotmail.com

${ }^{4}$ MD, Endocrinóloga. Pontificia Universidad Javeriana, Hospital Universitario San Ignacio. Unidad de Endocrinología. Bogotá, Colombia.cmgg77@hotmail.com

${ }^{5} \mathrm{MD}$, Endocrinólogo. Hospital Erasmo Meoz. Clínica San José, Cúcuta, Colombia.freddynino51@hotmail.com

${ }^{6}$ MD, Endocrinólogo. Clínica de Tiroides. Clínica Comfamiliar. Universidad Tecnológica de Pereira. Torre 1 consultorio 408,

Pereira, Colombia.hmaq@hotmail.com

${ }^{7} \mathrm{MD}$, Endocrinólogo. Profesor Asociado Universidad Industrial de Santander UIS, Hospital Universitario de Santander. Bucaramanga, Colombia. heavenlycouott@hotmail.com

${ }^{8} \mathrm{MD}$, Endocrinólogo. San Vicente Fundación Centros

Especializados- Clínica Somer. Rionegro, Antioquia, Colombia. nlpendocrino@gmail.com

${ }^{9}$ MD, Endocrinólogo. Clínica de la Presentación - Hospital Departamental Santa Sofía. Manizales, Colombia.dadelapo@ gmail.com

${ }^{10}$ MD, Endocrinóloga. Fundación Valle del Lili. Cali, Colombia. kaferiz@gmail.com

${ }^{11} \mathrm{MD}$, Endocrinólogo. ENDHO Colombia. Neiva, Colombia. alepyto@yahoo.com.

${ }_{12} \mathrm{MD}$, Cirujano Endocrino. Hospital Pablo Tobón Uribe. Instituto de Cancerología Las Américas. Medellín, Colombia.jpduenas@ cirendocrina.com
${ }^{13}$ MD, Endocrinólogo. Universidad Libre, Cali. Centro Médico Imbanaco. Cali, Colombia. alinabreu@imbanaco.com.co

${ }^{14} \mathrm{MD}$, Endocrinólogo. MSc Oncología Molecular. Servicio de Endocrinología Oncológica. Instituto Nacional de Cancerología. Bogotá, Colombia.ffierro@cancer.gov.co

${ }^{15} \mathrm{MD}$, Endocrinólogo. Centro Médico Bucaramanga. Bucaramanga, Colombia.jbpbmd@gmail.com

${ }^{16} \mathrm{MD}$, Endocrinólogo. Clínica Las Américas. Endocrino Centro de Estudios Hormonales S.A. Medellín, Colombia. jotatorres@gmail. com

${ }^{17} \mathrm{MD}$, Endocrinólogo. Universidad Pontificia Bolivariana, Clínica Integral de Diabetes. Medellin, Colombia. palacioendocrino@gmail. com

${ }^{18}$ MD, Fellow en Medicina Nuclear. SPECT Medicina Nuclear. Clínica Carlos Ardila Lulle. Floridablanca, Santander, Colombia. lizethsanchezorduz@gmail.com

${ }^{19}$ MD, Médico Nuclear. SPECT Medicina Nuclear. Clínica Carlos Ardila Lulle. Floridablanca, Santander, Colombia. ragarey60@ gmail.com. (Autor fallecido)

\section{Autor de correspondencia}

Edwin Antonio Wandurraga Sánchez

Carrera 24 \#154-106, Centro médico Carlos Ardila Lulle, Torre B, Piso 8, módulo 55, consultorio 806. Floridablanca, Santander. edwinwandurraga@gmail.com.

Fecha de recepción: 19/09/2018

Fecha de aceptación: 31/10/2018

\section{Resumen}

Introducción: Los registros poblacionales de cáncer son una aproximación útil para determinar la magnitud del problema, pero son insuficientes como fuente de variables de 
interés para los clínicos y como generadores de hipótesis de investigación. Con el objetivo de identificar las características clínicas de los pacientes con cáncer de tiroides, el enfoque terapéutico inicial y los costos asociados con el manejo de la enfermedad, se implementó un registro específico en 10 ciudades de Colombia.

Materiales y métodos: Se llevó a cabo un estudio descriptivo, observacional entre los años 2013 y 2015. Se desarrolló una herramienta web (www.colombiatiroides.com) consistente en 55 variables con características demográficas, histopatológicas, paraclínicas y terapéuticas, para registrar la información de los pacientes con diagnóstico de cáncer de tiroides que asistieron a consulta de endocrinología, medicina nuclear, cirugía de cabeza y cuello o cirugía endocrina.

Resultados: Se incluyó información de 1.096 pacientes con cáncer de tiroides. El promedio de edad fue de 45,5 años; 86,3\% eran mujeres; $93,6 \%$ de los casos correspondieron a carcinoma papilar. Se documentó asociación entre el tamaño tumoral y el compromiso capsular, extracapsular, linfovascular y ganglionar central y lateral $(\mathrm{p}=0,000)$. Los pacientes menores de 45 años presentaron mayor invasión ganglionar central $(p=0,000)$ y lateral $(p=0,003)$, mientras que en los mayores de 45 años los tumores multicéntricos $(p=0,032)$ y la infiltración extracapsular $(\mathrm{p}=0,036)$ fueron más frecuentes. El costo directo aproximado por paciente/año fue de \$2'532.687 (982 USD).

Conclusiones: Los resultados de este registro muestran que el carcinoma diferenciado de tiroides de tipo papilar corresponde al 93,6\% dentro de todos los tipos de cáncer de tiroides, proporción que es mayor a la de otras series reportadas en la literatura. El 89\% de los pacientes con carcinoma diferenciado de tiroides fueron clasificados como de riesgo alto e intermedio de recurrencia, luego de la terapia inicial del cáncer de tiroides. En pacientes menores de 45 años, la enfermedad tiende a invadir por vía linfática, pero en los mayores de 45 años las extensiones locales son más frecuentes. Finalmente, el costo aproximado de la enfermedad es de USD 982 paciente/año.

\section{Abstract}

Background: Cancer registries are useful to determine the magnitude of the problem, but not enough to provide meaningful variables for clinicians or to serve as a source of hypotheses for research. Our objective was to identify the clinical characteristics of thyroid cancer patients, initial therapeutic approach and costs associated with the management of the disease.

Methods: A descriptive, observational case series study with thyroid cancer patients was conducted (2013 - 2015) in 10 cities in Colombia. A web-based tool (www.colombiatiroides.com) was developed to include patient data (55 variables, including demographic, histopathological, paraclinical and therapeutic characteristics).
Results: Information of 1,096 patients with thyroid cancer was included (Mean age: 45.5 years; $86.3 \%$ women). 93.6\% of cases were papillary carcinoma. Association between tumour size and capsular, extracapsular, lymphovascular, central and lateral lymph node involvement was documented $(p=0.000)$. Patients under 45 years showed greater central $(p=0.000)$ and lateral ( $p=0.003$ ) lymph node invasion while in patients over 45 , multifocal tumours ( $p=0.032$ ) and extracapsular infiltration ( $p=0.036)$ were more frequent. The approximate direct cost $/ p a$ tient/year was COP\$2,532,687 (USD 982).

Conclusions: This registry shows that papillary thyroid carcinoma accounts for $93.6 \%$ of all types of thyroid cancer, a higher percentage than reported in other series in the literature; $89 \%$ of patients were classified as high and intermediate risk of recurrence following initial cancer therapy. In patients under 45 years, the disease tends to disseminate via the lymphatic system, but in patients over 45 local extensions are more frequent. Finally, approximate disease cost was estimated at USD 982/patient/year.

Palabras clave: cáncer de tiroides, registro, patología, tratamiento, costos, epidemiología.

Key words: thyroid cancer, registry, pathology, treatment, costs, epidemiology.

\section{Introducción y objetivo}

El cáncer de tiroides es la neoplasia endocrina más frecuente y su incidencia ha aumentado progresivamente en las últimas décadas ${ }^{(1)}$. De acuerdo con la información reportada en GLOBOCAN, en el año 2012 se registraron a nivel mundial 67.179 nuevos casos de esta patología oncológica en hombres y 229.923 en mujeres, con una incidencia de 1,9 y 6,1 por 100.000 habitantes, respectivamente ${ }^{(2)}$. Esta enfermedad explica aproximadamente el 1,5\% del número total de nuevos casos de cáncer para todos los sitios anatómicos y un 0,4\% del número total de muertes relacionadas con cáncer, por año ${ }^{(3,4)}$.

El Registro Poblacional de Cáncer en Colombia, (20062010), estimó una incidencia de cáncer de tiroides de 2,9 casos/100.000 personas año en hombres y 13,1 casos/100.000 personas año en mujeres ${ }^{(5)}$. Si bien los registros son una aproximación útil para determinar la magnitud de un problema, no son siempre una fuente adecuada de variables de interés para los clínicos y son pobres generadores de hipótesis e ideas de investigación. En el trabajo realizado por Cendales y cols. se evaluó la calidad de la información de cuatro registros de incidencia de cáncer en Colombia, y se encontró un subregistro importante. Ninguno de los registros evaluó a profundidad variables clínicas, terapéuticas o de $\operatorname{costos}^{(6)}$.

Con el objetivo de identificar las características clínicas, el enfoque terapéutico inicial de los pacientes con cáncer de tiroides y los costos asociados al manejo de la enfermedad en Colombia, se registró, en una herramienta web, la información relacionada con 55 variables específicas de los pacientes. 


\section{Materiales y métodos}

Se realizó un estudio descriptivo observacional, tipo serie de casos, entre enero del 2013 y enero del 2015, en 10 ciudades de Colombia (Bogotá, Medellín, Rionegro, Bucaramanga, Cali, Cúcuta, Manizales, Neiva, Pereira y San Andrés).

El estudio incluyó 1.096 registros de pacientes con cáncer de tiroides. La información fue consignada por 19 médicos $(14$ endocrinólogos, 2 especialistas en medicina interna, 2 especialistas en medicina nuclear y un cirujano endocrino), en 12 centros médicos ( 9 privados y 3 públicos).

El estudio incluyó todos los pacientes con diagnóstico de cáncer de tiroides que asistieron a consulta de endocrinología y cirugía endocrina de los 19 médicos que participaron en el estudio. La información fue registrada utilizando una herramienta web diseñada por los autores para la cuantificación específica de las características epidemiológicas, clínicas y de impacto de costos de las neoplasias malignas de tiroides en este país. Esta plataforma en línea fue creada en PHP, Apache y MySQL. El consentimiento informado de los pacientes no fue requerido para este estudio, y el registro fue aprobado por el Comité de Ética del Hospital Universitario San Ignacio en Bogotá.

El estudio consideró variables demográficas (fecha de nacimiento, sexo, tipo de vinculación al sistema de salud y ciudad en la que se administra el tratamiento), características histopatológicas (tipo y variante histológica, tamaño tumoral, compromiso multicéntrico, capsular, extracapsular, linfovascular y ganglionar y clasificación TNM). Las variables clínicas y terapéuticas incluyeron características del procedimiento quirúrgico, terapia con yodo radiactivo, hallazgos del rastreo posterapia ablativa, tratamiento farmacológico y resultados de exámenes de laboratorio e imágenes diagnósticas.

Así mismo, en el sistema de registro se incluyeron también variables sobre los valores de los últimos exámenes paraclínicos (TSH, tiroglobulina y anticuerpos antitiroglobulina).

\section{Determinación de los costos}

Se realizó una estimación a los costos directos del tratamiento integral de los 1.096 pacientes en un periodo de 12 meses, de acuerdo con el número de eventos quirúrgicos, dosis de yodo recibidas, frecuencia de realización de exámenes de laboratorio e imágenes diagnósticas y tratamiento farmacológico. Se incluyeron dentro de la estimación del costo de tratamiento del cáncer de tiroides el número de veces que se realizaron los exámenes de laboratorio en el último año, y la dosis requerida de yodoterapia (costos directos).También se incluyó el tratamiento farmacológico que recibieron los pacientes.

Las tarifas se obtuvieron del manual tarifario ISS $2001^{(19)}$. Para el cálculo del paquete quirúrgico se sumó al costo del procedimiento según ISS 2001 (Manual de precios de la Seguridad Social 2001) un 28\% adicional ${ }^{(19)}$. Para los procedimientos para los cuales no había una tarifa de referencia ISS 2001 (resonancia nuclear magnética de cuello y tomografía
Tabla 1. Características clínicas e histopatológicas en 1.096 registros de pacientes con diagnóstico de cáncer de tiroides en Colombia

\begin{tabular}{|c|c|c|}
\hline Característica & $\begin{array}{l}\text { Número de } \\
\text { casos con } \\
\text { variable de } \\
\text { interés }(n)\end{array}$ & $\begin{array}{c}\text { Casos } \\
(\%)\end{array}$ \\
\hline $\begin{array}{l}\text { Edad } \\
<45 \text { años } \\
\geq 45 \text { años }\end{array}$ & 1.027 & $\begin{array}{l}453(44,1) \\
574(55,8)\end{array}$ \\
\hline $\begin{array}{l}\text { Sexo } \\
\text { Mujeres } \\
\text { Hombres }\end{array}$ & 1.096 & $\begin{array}{l}946(86,4) \\
150(13,6)\end{array}$ \\
\hline $\begin{array}{l}\text { Tipo de cáncer } \\
\text { Papilar } \\
\text { Folicular } \\
\text { Hürthle } \\
\text { Medular } \\
\text { Anaplásico }\end{array}$ & 1.096 & $\begin{array}{c}1.026 \\
(93,6) \\
37(3,4) \\
14(1,3) \\
19(1,7) \\
0 \\
\end{array}$ \\
\hline $\begin{array}{l}\text { Variante histológica Ca papilar } \\
\text { Clásica } \\
\text { Folicular } \\
\text { Célula alta } \\
\text { Esclerosante } \\
\text { Otros }\end{array}$ & 925 & $\begin{array}{c}615(66,4) \\
225(24,3) \\
57(6,1) \\
11(1,1) \\
17(1,8)\end{array}$ \\
\hline $\begin{array}{l}\text { Tamaño } \\
\leq 1 \mathrm{~cm} \\
1-4 \\
\geq 4 \mathrm{~cm}\end{array}$ & 951 & $\begin{array}{l}256(26,9) \\
560(58,8) \\
135(14,1)\end{array}$ \\
\hline Multifocalidad & 984 & $375(38,1)$ \\
\hline Compromiso de la cápsula tiroidea & 954 & $522(54,7)$ \\
\hline $\begin{array}{l}\text { Compromiso del tejido } \\
\text { extracapsular }\end{array}$ & 928 & $299(32,2)$ \\
\hline Invasión linfovascular & 895 & $321(35,8)$ \\
\hline Realización de vaciamiento central & 1.092 & $709(64,9)$ \\
\hline $\begin{array}{l}\text { Compromiso tumoral de ganglios } \\
\text { del compartimento central }\end{array}$ & 872 & $447(51,2)$ \\
\hline Realización de vaciamiento lateral & 1.092 & $175(16)$ \\
\hline $\begin{array}{l}\text { Compromiso tumoral de ganglios } \\
\text { del compartimento lateral }\end{array}$ & 758 & $178(23,4)$ \\
\hline
\end{tabular}

por emisión de positrones PET-CT) se calculó un valor aproximado a partir de cotizaciones en dos centros nacionales de referencia de imágenes diagnósticas. El costo de la administración de yodo se calculó a partir de la tarifa básica aplicada en la unidad de medicina nuclear SPECT LTDA. (Ver material suplementario). 


\section{Análisis estadístico}

Se realizó un análisis descriptivo mediante el software STATA VE 12.0 y se consideró un nivel de significancia del 5\%. Se realizaron comparaciones de variables mediante pruebas chi-cuadrado y el test exacto de Fisher, según fuese apropiado.

\section{Resultados}

\section{Variables demográficas}

De los 1.096 pacientes, 946 (86,3\%) eran mujeres, con una relación mujer/hombre de 6:1. La media de edad de los pacientes al momento del diagnóstico fue de 45,5 \pm 14 años. La edad, de acuerdo con el sexo, al momento de hacer el diagnóstico de cáncer de tiroides, mostró una mayor proporción de hombres mayores de 45 años, en comparación con mujeres $(62,5 \%$ vs. $51,6 \%, p=0,017)$. La tabla 1 muestra la frecuencia de las características demográficas e histopatológicas más relevantes.

\section{Variables histopatológicas}

El carcinoma papilar fue el tipo de cáncer de tiroides más frecuentemente reportado $(93,6 \%)$, seguido por el carcinoma folicular $(3,3 \%)$, el carcinoma medular $(1,7 \%)$ y el carcinoma

Tabla 2. Clasificación TNM y estadio en 1.096 registros de pacientes con diagnóstico de cáncer de tiroides en Colombia

\begin{tabular}{|c|c|c|}
\hline Característica & $\begin{array}{c}\text { Número de } \\
\text { casos con la } \\
\text { variable de } \\
\text { interés }\end{array}$ & Casos (\%) \\
\hline \multicolumn{3}{|l|}{ Clasificación TNM } \\
\hline Tamaño (T) & 1.096 & \\
\hline $\mathrm{T} 1$ & & $415(37,8)$ \\
\hline $\mathrm{T} 2$ & & $201(18,3)$ \\
\hline T3 & & $303(27,6)$ \\
\hline T4 & & $81(7,3)$ \\
\hline Tx & & $96(87,5)$ \\
\hline Estado nodal (N) & 1.096 & \\
\hline No & & $446(40,6)$ \\
\hline $\mathrm{N} 1 \mathrm{a}$ & & $293(26,7)$ \\
\hline $\mathrm{N} 1 \mathrm{~b}$ & & $203(18,5)$ \\
\hline $\mathrm{Nx}$ & & $154(14,0)$ \\
\hline Metástasis a distancia (M) & 1.096 & \\
\hline MO & & $980(89,4)$ \\
\hline M1 & & $62(5,6)$ \\
\hline $\mathrm{Mx}$ & & $54(4,9)$ \\
\hline Estadio & 965 & \\
\hline I & & $587(53,5)$ \\
\hline II & & $78(7,1)$ \\
\hline III & & $168(15,3)$ \\
\hline IV & & $132(13,6)$ \\
\hline
\end{tabular}

de células de Hürthle (1,2\%). No hubo diferencia en la distribución del tipo de cáncer según el sexo. $(p=0,71)$.

Respecto a la variedad histológica del cáncer papilar, el $66.4 \%$ correspondió a la variante clásica, seguido por la variante folicular en el $24,3 \%$ de los pacientes y de célula alta en el $6.1 \%$. Se reportaron 11 casos con la variedad esclerosante difusa (3,7\%). En 127 casos (12,3\%) se reportaron al menos dos variantes en una misma pieza quirúrgica. La media del tamaño tumoral fue de 2,2 $\pm 1,62 \mathrm{~cm}$, con un rango entre 0,2 y $11 \mathrm{~cm}$. Doscientos cincuenta y seis casos $(26,9 \%)$ fueron microcarcinomas. El tamaño promedio del tumor fue de $28,6 \mathrm{~mm}$ en hombres y $20,9 \mathrm{~mm}$ en mujeres $(p=0,001)$. La tabla 2 muestra la clasificación TNM y el estadio de los pacientes (1.096 registros). En relación con el tamaño del tumor y la asociación potencial con otras variables, se encontró una relación directa entre el tamaño del tumor y el compromiso capsular y la invasión linfovascu$\operatorname{lar}(p=0,00)$. De la misma manera, se encontró asociación entre el tamaño del tumor y un mayor compromiso extracapsular y de los nódulos linfáticos centrales y laterales $(p=0,00)$.

La tabla 3 muestra el resultado del análisis grupal de sujetos mayores y menores de 45 años, y de los diversos hallazgos

Tabla 3. Análisis de las variables histopatológicas según el grupo de edad

\begin{tabular}{|c|c|c|}
\hline & $\begin{array}{l}\text { Número } \\
\text { de casos/ } \\
\text { número de } \\
\text { registros (\%) }\end{array}$ & Valor de $p$ \\
\hline $\begin{array}{l}\text { Compromiso de la cápsula } \\
<45 \text { años } \\
\geq 45 \text { años }\end{array}$ & $\begin{array}{l}216 / 409(52,8) \\
289 / 495(58,3)\end{array}$ & 0,093 \\
\hline $\begin{array}{l}\text { Compromiso extracapsular } \\
<45 \text { años } \\
\geq 45 \text { años }\end{array}$ & $\begin{array}{l}116 / 397(29,2) \\
172 / 479(35,1)\end{array}$ & 0,036 \\
\hline $\begin{array}{l}\text { Invasión linfovascular } \\
<45 \text { años } \\
\geq 45 \text { años }\end{array}$ & $\begin{array}{l}148 / 376(39,3) \\
162 / 468(34,6)\end{array}$ & 0,155 \\
\hline $\begin{array}{l}\text { Multifocalidad global } \\
<45 \text { años } \\
\geq 45 \text { años }\end{array}$ & $\begin{array}{l}147 / 424(34,6) \\
211 / 508(41,5)\end{array}$ & 0,032 \\
\hline $\begin{array}{l}\text { Ganglios compartimento } \\
\text { central positivos para } \\
\text { malignidad } \\
<45 \text { años } \\
\geq 45 \text { años }\end{array}$ & $\begin{array}{l}240 / 391(61,3) \\
185 / 438(42,2)\end{array}$ & 0,000 \\
\hline $\begin{array}{l}\text { Ganglios compartimento } \\
\text { lateral positivos para } \\
\text { malignidad } \\
<45 \text { años } \\
\geq 45 \text { años }\end{array}$ & $\begin{array}{l}94 / 336(27,9) \\
72 / 384(18,7)\end{array}$ & 0,003 \\
\hline
\end{tabular}


histopatológicos conocidos para predecir recurrencia tumoral. En pacientes menores de 45 años con información acerca del compromiso de los nódulos linfáticos, el 61,3\% fueron positivos para compromiso central y el $27,9 \%$ para compromiso lateral vs. $42,2 \%$ y $18,7 \%$ en pacientes mayores de 45 años, respectivamente $(p=0.000)$.

Adicionalmente, se reportó compromiso extracapsular en el $35,1 \%$ de los registros en sujetos mayores de 45 años, y en $29,2 \%$ de aquellos menores de 45 años ( $\mathrm{p}=0,0036)$. Se evaluó también la asociación entre sexo y factores predictores de recaída (tabla 4).

Tabla 4. Análisis de las variables histopatológicas h según el género

\begin{tabular}{|c|c|c|}
\hline & $\begin{array}{l}\text { Número de } \\
\text { casos/ } \\
\text { Número de } \\
\text { registros (\%) }\end{array}$ & Valor de $p$ \\
\hline $\begin{array}{l}\text { Compromiso de la cápsula } \\
\text { Masculino } \\
\text { Femenino }\end{array}$ & $\begin{array}{c}82 / 131(62,6) \\
440 / 823(53,4)\end{array}$ & 0,051 \\
\hline $\begin{array}{l}\text { Compromiso extracapsular } \\
\text { Masculino } \\
\text { Femenino }\end{array}$ & $\begin{array}{c}52 / 126(41,2) \\
247 / 802(30,7)\end{array}$ & 0,019 \\
\hline $\begin{array}{l}\text { Invasión linfovascular } \\
\text { Masculino } \\
\text { Femenino }\end{array}$ & $\begin{array}{l}47 / 122(38,5) \\
274 / 773(35,4)\end{array}$ & 0,51 \\
\hline $\begin{array}{l}\text { Multifocalidad global } \\
\text { Masculino } \\
\text { Femenino }\end{array}$ & $\begin{array}{c}59 / 134(44,0) \\
316 / 850(37,1)\end{array}$ & 0,129 \\
\hline $\begin{array}{l}\text { Ganglios compartimento } \\
\text { central positivos para } \\
\text { malignidad } \\
\text { Masculino } \\
\text { Femenino }\end{array}$ & $\begin{array}{c}71 / 115(61,7) \\
376 / 757(49,6)\end{array}$ & 0,016 \\
\hline $\begin{array}{l}\text { Ganglios compartimento } \\
\text { lateral positivos para } \\
\text { malignidad } \\
\text { Masculino } \\
\text { Femenino }\end{array}$ & $\begin{array}{l}37 / 101(36,6) \\
141 / 657(21,4)\end{array}$ & 0,001 \\
\hline
\end{tabular}

\section{Variables terapéuticas}

De los 1.070 registros en los que se describió el tipo de procedimiento quirúrgico, el 94,2\% correspondían a tiroidectomía total, de las cuales el $26 \%$ fueron realizadas por cirujanos generales, $11 \%$ por cirujanos oncólogos, y $9 \%$ por cirujanos endocrinólogos. Se hizo disección de los nódulos linfáticos centrales en el $64,9 \%$ de los pacientes. De acuerdo con la clasificación TNM, el 37,8\% de los reportes fueron T1, 18,3\% T2,y
$34,9 \%$ T3 y T4. Se realizó vaciamiento lateral en el 16\% de los pacientes (tabla 1).

Por otra parte, se administró terapia con yodo radiactivo a 885 pacientes $(86,5 \%)$. Se administró una dosis a 712 pacientes $(80,4 \%)$. A 81 pacientes se les administraron dos dosis $(9,8 \%)$. Se encontró una media de duración de 6,6 meses entre la cirugía y la administración del ${ }^{131}$. La dosis promedio de yodo fue de 120,3 mCi. La dosis promedio administrada a los pacientes clasificados como de bajo riesgo $(n=106)$ fue de $86,4 \mathrm{mCi}$ y en los de riesgo intermedio o alto $(n=776)$ fue de $123 \mathrm{mCi}$. De los 90 pacientes con microcarcinoma unifocal, sin características de riesgo de recurrencia, 34 pacientes $(37,7 \%)$ recibieron terapia ablativa con yodo.

Referente a los hallazgos en el rastreo posterapia ablativa se encontró remanente tiroideo en el 83,8\%, metástasis locorregionales en el 6,3\% y metástasis a distancia en el 6,6\% de los registros, de los cuales el 94,7\% tenían compromiso pulmonar. En el 10\% de las terapias ablativas se utilizó TSH recombinante, como preparación; el 1,7\% de la población recibió radioterapia externa.

Respecto del tratamiento farmacológico se reportó tratamiento con levotiroxina en 1.092 registros $(99,6 \%)$ con una dosis promedio de $146 \pm 54,2 \mathrm{mcg}$. El uso de carbonato de calcio + vitamina D se reportó en el $42,3 \%$ de los pacientes con una mediana de dosis de $1.200 \mathrm{mg} /$ día. El 20,5\% de los pacientes recibían calcitriol en el momento del registro con una mediana de $0,5 \mathrm{mcg} /$ día. Por último, seis pacientes se encontraban recibiendo tratamiento con inhibidor de tirosina cinasa (sorafenib) con una dosis promedio de $540 \mathrm{mg}$. (400-800 mg).

Al evaluar la necesidad de hospitalización atribuida al cáncer de tiroides o a su tratamiento en los últimos 12 meses previos al registro, se reportó ingreso hospitalario en el 35,3\% de los pacientes con una mediana de duración de tres días. El 31,5\% de los pacientes fueron incapacitados en el último año, con una mediana de duración de 10 días (rango entre 1 y 365 días).

\section{Costos directos}

El costo aproximado del tratamiento de 1.096 pacientes con cáncer de tiroides en un año de seguimiento, independientemente del momento quirúrgico inicial, fue de \$2'772.826 (955 USD) y el costo aproximado por paciente/año se estimó en \$2'532.687 (982 USD, TRM mayo 2016). En la tabla 5 se muestra la distribución de los costos según el tipo de intervención. No se realizó análisis de costos indirectos.

\section{Discusión}

Aunque este estudio de serie de casos no buscaba evaluar ni la incidencia ni la prevalencia del cáncer de tiroides en Colombia, constituye una muestra representativa de la situación actual de la enfermedad en el país. 
Tabla 5. Costos directos del tratamiento de 1096 pacientes con cáncer de tiroides en 12 meses de seguimiento

\begin{tabular}{l|c|c|c}
\hline Tipo de intervención & $\begin{array}{c}\text { \# pacientes con registro de la } \\
\text { intervención }\end{array}$ & $\begin{array}{c}\text { Costo total de la intervención } \\
\text { según número de eventos* }\end{array}$ & $\begin{array}{c}\text { Costo } \\
\text { paciente / año* }\end{array}$ \\
\hline Terapia farmacológica & 1096 & 505.783 & 461,4 \\
\hline Laboratorios & 833 & 54.206 & 49,4 \\
\hline Imágenes diagnósticas & 724 & 35.505 & 32,3 \\
\hline Terapia con I131 & 153 & 226.921 & 207 \\
\hline Procedimiento & 197 & 232.315 & 212 \\
\hline quirúrgico & 17 & 21.414 & 19,5 \\
\hline TSH recombinante & & $\mathbf{1} \mathbf{0 7 6 . 1 4 4}$ & $\mathbf{9 8 2}$ \\
\hline
\end{tabular}

* USD

El análisis de esta serie de 1.096 registros reafirma la mayor frecuencia de presentación del cáncer de tiroides en el sexo femenino, siendo la relación mujer/hombre (6:1) superior a la reportada por el registro SEER de forma global (3:1) y en población hispana $(4: 1)^{(7)}$. Este hecho podría explicarse por una mayor frecuencia de consulta a los servicios de salud por parte de la población femenina en Colombia y/o a un posible papel de los estrógenos en la patogenia de la enfermedad ${ }^{(8-10)}$. Por otra parte, la curva de distribución de los casos de acuerdo con la edad fue similar a la serie americana, con un pico de presentación entre los 40 y los 60 años $^{(8)}$.

En cuanto al tipo de tumor, la proporción del carcinoma papilar de tiroides fue mayor a la reportada en países como Brasil o Estados Unidos ${ }^{(11)}$ (93,6\% de los registros), mientras que la prevalencia del carcinoma folicular fue muy baja. Este hallazgo puede estar relacionado con la conocida sobrecorrección de la deficiencia de yodo en Colombia ${ }^{(12)}$ ya que, como se ha descrito, el carcinoma folicular tiende a presentarse con menor frecuencia en zonas con suficiencia de yodo ${ }^{(13)}$.

En este estudio se encontró que el grupo de pacientes menores de 45 años presentó mayor compromiso ganglionar central y lateral. Por otro lado, los pacientes de 45 o más años tuvieron un mayor compromiso extracapsular y multifocal. Lo anterior sugiere que, mientras los tumores de los pacientes jóvenes tienen la capacidad de invadir a través de los vasos linfáticos, en los mayores de 45 años tienden a invadir por continuidad, mostrando una habilidad biológica para atravesar membranas. El mayor tamaño tumoral se asoció con el sexo masculino, con compromiso extracapsular y ganglionar central y lateral, sugiriendo que existe de un acceso tardío a los servicios de salud en esta población.

La tiroidectomía total sigue siendo el tratamiento quirúrgico de elección en el cáncer de tiroides, realizándose en el $94,2 \%$ de los pacientes. Tres de cada 4 fueron ejecutadas por un cirujano especialista.

En el 51,2\% del total de los registros se encontró compromiso ganglionar central. Esta proporción disminuyó al 33,2\% en el subgrupo de microcarcinomas. Este dato es similar a lo encontrado en la serie de la Clínica Mayo, en 900 pacientes en un período mayor a 60 años ${ }^{(14)}$, e inferior a lo reportado por Ito y colaboradores en un estudio japonés con más de 2.400 casos, en el que se encontró compromiso ganglionar central en el $57 \%$ de los vaciamientos ${ }^{(15)}$.

Se realizó vaciamiento central en el 60,5\% de los pacientes con microcarcinoma, lo que sugiere una tendencia entre los cirujanos locales a realizar vaciamiento central profiláctico, hecho que ha causado una controversia acerca de la práctica de vaciamiento central de rutina como parte del tratamiento inicial de los microcarcinomas. Adicionalmente se encontró un sobreuso de terapia con yodo radiactivo en pacientes con microcarcinoma. Uno de cada tres pacientes con tumor $<1$ $\mathrm{cm}$, sin otros factores de riesgo, recibió terapia ablativa. Estos hallazgos sugieren la necesidad de evaluar la adherencia a las guías de práctica clínica.

Al comparar esta serie con la publicada por Mazzaferri en el año $2000^{(16)}$, se encuentra un número similar de hallazgos de remanente tiroideo en el rastreo posterapia con yodo radiactivo (83,8 vs $87 \%$ ) y un significativo menor compromiso metastásico a nivel locorregional $(6,3 \%$ vs $36 \%)$ y a distancia $(6,6 \%$ vs $13 \%)$. Este hallazgo podría explicarse por una detección más temprana del cáncer de tiroides, producto del sobreuso de métodos diagnósticos como la ecografía, y a una más frecuente realización de vaciamiento central rutinario como parte de la terapia inicial.

De acuerdo a la guía de la Asociación Americana de Tiroides $\left(\right.$ ATA ${ }^{(17)}$ el $22,8 \%$ de los pacientes tenían alto riesgo de recurrencia, el $67 \%$ riesgo intermedio y el $10,2 \%$ riesgo bajo. Este estudio no registró variables adicionales tales como, la extensión del compromiso ganglionar y el estado mutacional. En contraste, según el sistema de clasificación de riesgo de la Asociación Latinoamericana de Tiroides (LATS), el 89\% de los pacientes se encontraban en el grupo de alto riesgo. En comparación con el estudio realizado en Argentina por Pitoia ${ }^{(18)}$, los resultados de este registro muestran un mayor porcentaje 
de pacientes en categoría de riesgo alto (22\% vs. $17 \%$ ) e intermedio (67\% vs. $48 \%$ ) según ATA, y en categoría de riesgo alto ( $89 \%$ vs. $71 \%$ ) según LATS, lo que obliga a un seguimiento riguroso en nuestra población.

En esta serie, el promedio de costo directo de manejo de la enfermedad, paciente/año, fue de COP 2'532.687 (982 USD, TRM mayo 2016). Es importante enfatizar en que esta es la primera vez que se analiza el impacto económico del cáncer de tiroides en Colombia, en términos actuales y representativos.

Teniendo en cuenta el tipo de estudio y la falta de variables tales como la tiroglobulina estimulada o el seguimiento de la tiroglobulina, este estudio no define o clasifica la respuesta inicial al tratamiento.

En conclusión, de acuerdo con los resultados de este registro, el carcinoma papilar de tiroides representa el 93,6\% de todos los tipos de cáncer de tiroides en Colombia, mientras que el carcinoma folicular es raro. De todos los pacientes con cáncer de tiroides diferenciado, el 89\% se clasificó como de riesgo alto e intermedio de recurrencia, luego de la terapia inicial para el cáncer de tiroides, creando la necesidad de un estrecho seguimiento. Se encontró también que, mientras que la enfermedad tiende a invadir más frecuentemente a través del sistema linfático en pacientes menores de 45 años, la extensión local de la enfermedad es más frecuente en los pacientes mayores de 45 años. Con base en la información derivada de este registro, el costo aproximado de la enfermedad fue estimado en USD 982 paciente/año. Finalmente, el uso de herramientas web para implementar registros se considera como una buena estrategia para obtener una muestra representativa e identificar el comportamiento de una enfermedad específica.

\section{Agradecimientos}

Agradecimiento especial a Pradilla LP, Folleco M, Parra GA, Restrepo LM, Palacios KL, Stephens I, Arana I, Buendía R y Gómez DF, por la contribución en registros de pacientes. A Rondón M, por liderar el analisis estadístico y a Integralis HGS financiado por Genzyme Colombia Ltda., por la asesoría editorial y de escritura en la preparación de este manuscrito.

\section{Fuentes de financiación}

Ninguna. Los autores no recibieron recursos de financiación. Se recibió soporte en edición y escritura para la preparación del manuscrito por parte de Integralis HGS financiado por Genzyme de Colombia Ltda., pero lo autores fueron completa- mente responsables de todo el contenido y de las decisiones de edición del manuscrito.

\section{Conflictos de interés}

Los autores declaran que no tienen intereses personales, políticos, académicos o comerciales.

Material suplementario. Tabla de costos según tipo de intervención

\begin{tabular}{l|c}
\hline \multicolumn{1}{c}{ Tipo de intervención } & Costo* \\
Medicamentos & \\
\hline Levotiroxina (1 mcg) & 0,00089 \\
Carbonato de calcio + Vitamina D (1 mg) & 0,0007 \\
Calcitriol (1 mcg) & 1,05 \\
Sorafenib (1 mg) & 0,13 \\
\hline Laboratorios & \\
\hline TSH & 9,3 \\
Tiroglobulina & 13,8 \\
Anticuerpos antitiroglobulina & 14,1 \\
\hline Imágenes diagnósticas & \\
\hline Ecografía de tiroides & 9,3 \\
Tomografía axial computarizada de tórax & 39,1 \\
Resonancia nuclear magnética de cuello & 228 \\
PET CT & 852 \\
\hline Terapia con yodo 131 & 1.155 \\
\hline 1 mCi & 1.649 \\
Terapia para cáncer & 12,6 \\
Rastreo posterapia & 165,6 \\
TSH recombinante & 63,9 \\
\hline Intervención quirúrgica & 1.260 \\
\hline Hemitiroidectomía & \\
Tiroidectomía total (TT) & 775 \\
Vaciamiento central (VC) & \\
Vaciamiento unilateral de cuello (VLU) & \\
Vaciamiento bilateral de cuello (VLB) & \\
TT+VC & \\
\hline
\end{tabular}

* USD (basado en referencia 19) 


\section{Referencias}

1. Cooper DS, Doherty GM, Haugen BR, Kloos RT, Lee SL, Mandel SJ, et al. Revised American Thyroid Association management guidelines for patients with thyroid nodules and differentiated thyroid cancer. American Thyroid Association (ATA) Guidelines Taskforce on Thyroid Nodules and Differentiated Thyroid Cancer. Thyroid. 2009 Nov;19(11):1167-214.

2. GLOBOCAN 2012. Estimated cancer incidence, mortality and prevalence worldwide in 2012. World Health Organization. http://globocan.iarc.fr/

3. Jemal A, Siegel R, Ward E, Hao Y, Xu J, Thun MJ. Cancer statistics, 2009. CA Cancer J Clin. 2009; 59: 225-249.

4. Gagel RF, Goepfert H, Callender DL. Changing concepts in the pathogenesis and management of thyroid carcinoma. CA Cancer J Clin 1996; 46: 261-283.

5. Garavito G, Llamas Olier A, Cadena E, De Los Reyes A, Hurtado G, Rojas L, et al. Manejo multidisciplinario del cáncer diferenciado de tiroides en el Instituto Nacional de Cancerología. Rev Colomb Cancerol. 2010; 14(2):65-77.

6. Cendales R, Pardo C, Uribe C, López G, Yepes MC, Bravo LE. Calidad de los datos en los registro de cáncer de base de población en Colombia. Biomédica. $2012 ; 32(4): 536-44$.

7. www.seer.cancer.gov/statfacts/html/thyro.html.

8. Xu S, Chen G, Peng W, Renko K, Derwahl M. Oestrogen action on thyroid progenitor cells: relevant for the pathogenesis of thyroid nodules? J Endocrinol 2013 Jun 1;218(1):125-33.

9. M, Goldner W, Beseler CL, Rogan EG, Cavalieri EL. Unbalanced estrogen metabolism in thyroid cancer. Int J Cancer. 2013 Dec 1;133(11):2642-9.

10. Rajoria S, Suriano R, Shanmugam A, Wilson YL, Schantz SP, Geliebter J, et al. Metastatic phenotype is regulated by estrogen in thyroid cells. Thyroid. 2010 Jan;20(1):33-41.

11. Veiga LH, Neta G, Aschebroo Kilfoy B, Ron E, Devesa SS. Thyroid cancer incidence patterns in Sao Paulo, Brazil and the US SEER program 1997-2008. Thyroid. 2013; 23(6:748-57).
12. Cuellar AA, Franco R, Valero MV. Desórdenes por deficiencia de yodo: Bocio endémico. Correlación entre la presencia de bocio y factores de crecimiento. Universidad Nacional de Colombia, Facultad de Medicina, Trabajo de grado. 2010

13. Petterson B, Adami ho, Wilander E, Coleman MP. Trends in thyroid cáncer incidence in Sweden, 1958-1981, by histopathologic type. Int J Cancer 1991; 48 (1):28.

14. Hay ID, Hutchinson ME, Gonzalez-Losada T, Mclver B, Reinalda ME, Grant CS, et al. Papillary thyroid microcarcinoma: a study of 900 cases observed in a 60-year period. Surgery 2008;144(6):980-7.

15. Ito Y1, Masuoka H, Fukushima M. Excellent prognosis of patients with solitary T1N0M0 papillary thyroid carcinoma who underwent thyroidectomy and elective lymph node dissection without radioiodine therapy. World Surg. 2010 Jun;34(6):1285-90.

16. Mazzaferri EL, Kloos RT. Clinical review 128: current approaches to primary therapy for papillary and folicullar thyroid cancer. J Clin Endocrino Metab. 2001;86(4):1447-63

17. Haugen BR, Alexander EK, Bible KC, Doherty GM, Mandel SJ, Nikiforov YE, et al. 2015 American thyroid association management guidelines for adult patients with thyroid nodules and differentiated thyroid cáncer. Thyroid. 2016 See comment in PubMed Commons below Jan;26(1):1-133.

18. Pitoia F, Ward L, Wohllk N, Friguglietti C, Tomimori E, Gauna A, et al. Recommendations of the Latin American Thyroid Society on diagnosis and management of differentiated thyroid cancer. Arq Bras Endocrinol Metabol. 2009 Oct; 53(7):884-7.

19. Acuerdo No. 256 DE 2.001. Por el cual se aprueba el "Manual de Tarifas" de la Entidad Promotora de Salud del Seguro Social "EPS-ISS". https://lexsaludcolombia.files.wordpress.com/2010/10/tarifas-iss-2001.pdf 